\title{
Sombras en el Enfoque Comunicativo
}

\section{Carmen de Castro Castro}

Dpto. de Lenguas romances, Estudios semíticos, Traducción e Interpretación.

Facultad de Ciencias de la Educación

Universidad de Córdoba

Recibido: 26 abril 2005 / Versión aceptada: 22 junio 2005

ISSN: $1697-7467$

\begin{abstract}
RESUMEN: La adopción, desde hace quince años, de un enfoque para la enseñanza del francés que primaba el uso de la lengua en el aula hacía prever que se conseguiría un adecuado dominio de este idioma extranjero. Sin embargo, el nivel de los alumnos ha descendido y el profesorado no ha asumido la nueva metodología.

Analizamos aquellos conceptos que, en su limitada aplicación actual, provocan, a nuestro juicio, un uso empobrecido de la lengua: situación de comunicación; enfoque discursivo; valor de los textos, gramática y vocabulario.

Subrayamos, así mismo, la falta de preparación del profesorado y las deficiencias programáticas de los libros de texto como factores fundamentales del escaso éxito obtenido por el enfoque comunicativo.

Palabras clave: Enfoque comunicativo, discurso, formación del profesorado, cultura francesa.
\end{abstract}

\begin{abstract}
The adoption, now fifteen years ago, of an approach to the teaching of French that took precedence over the use of the language in the classroom made us anticipate that an adequate command of this foreign language would be got. However, the level of the students has been lowered and teachers are not completely used to the new methodology.

We analyze the concepts that, in its limited actual application, cause (from our own viewpoint) an impoverished use of the language: communicative context, discourse approach, text function, grammar and vocabulary.

Similarly, we stress the lack of teaching training and the programme deficiencies found in textbooks as the fundamental responsible factors for the poor success of the Communicative approach.
\end{abstract}

Key words: Communicative approach, discourse, teaching training, french culture.

\section{INTRODUCCIÓN}

A veces, al tener que hacer mudanza en el despacho, aprovechamos para deshacernos de viejos artículos que amarillean en el archivador, y nos detenemos con agrado a releer aquellos que acogimos con entusiasmo y causaron nuestra admiración. Bérard, Porcher, Puren, Besse, Galisson, Bronckard, Cicurel, Coste; Grandcolas, Moirand, Pendanx, Peytard, Roulet o Vigner, eran protagonistas de la escuela francesa; Chaudron; Ellis, Long, Brumfit; Nunan, Widdowson... a los que, los menos conocedores de la lengua anglosajona, teníamos acceso. Nos movíamos 
entre el análisis inteligente y la contundencia de sus conceptos teóricos, disfrutando de la prosa y asombrándonos ante la coherencia de sus estudios.

Estas publicaciones sobre la enseñanza de las lenguas extranjeras de los años 80 y 90 , en las que alternaban las euforias con críticas precoces del enfoque comunicativo ${ }^{1}$, hacían prever una mejora sensible en el dominio de la lengua. Sus presupuestos y conceptos fundamentales $^{2}$ sirvieron para argumentar la necesidad de una Reforma y, sobre ellos, se construyeron los Diseños Curriculares de Lenguas Extranjeras y, últimamente, el Marco común europeo de referencia para las lenguas: aprendizaje, enseñanza, evaluación (MCERL).

Como no pocas veces en la historia de la metodología, la realidad del aula ha establecido la diferencia entre la teoría y la práctica y ha mostrado las grandes dificultades que sigue teniendo la implantación del Enfoque Comunicativo: mala comprensión y defectuoso seguimiento de los principios metodológicos; práctica escasa e ineficaz; resultados más que mediocres en el dominio de la lengua. Y, no obstante, el silencio se ha impuesto, como si hubiese dado su mejor fruto, pues callan la mayor parte de los profesionales de la enseñanza, abunda el negocio editorial y estremece la pasividad del alumnado que, al parecer, está satisfecho y logra comunicarse con fluidez en lengua extranjera.

\section{No TODO ES ENFOQUE COMUNICATIVO}

Sometidos en España a una fe uniforme en el enfoque dado a la enseñanza de la lengua ${ }^{3}$, parece haberse desdeñado otros criterios y prácticas, a pesar de que las metodologías que se basan en ellas hayan obtenido iguales o mejores resultados. No estaría fuera de lugar reconsiderar, por lo tanto, otras creencias y principios didácticos, tan válidos como los que sustentan el

${ }^{1}$ E. Bérard, (1995: 14) ya sostenía que l'A.C. n'a pas réussi à redéfinir la place de la composante linguistique et à rendre accessibles des démarches plus novatrices; L. Dabène, (1984: 45) defendía la realidad de la situación de clase en la que les échanges de classe relèvent de la métacommunication et le dialogue métacommunicatif non seulement est consubstantiel à l'acte éducatif par lequel on enseigne une langue non maternelle, mais en est l'aspect le plus fondamental. En cuanto a la focalización sobre el alumno, Ch. Puren afirmaba: La nécessité, en enseignement scolaire, d'un programme officiel de référence pour chaque classe, exclut ipso facto la possibilité d'une véritable centration constante sur chaque apprenant, comme l'exigerait l'interprétation maximaliste du concept. El mismo autor en 1995 concluye que: et l'approche communicative, bien qu'elle ait été en son temps développée par ses théoriciens et diffusée par ses formateurs comme une nouvelle "révolution méthodologique" impliquait en réalité l'abandon à terme de toute prétention à ce type de cohérence et le retour à une configuration 'éclectique.

${ }^{2}$ Le flou qui existe dans le cadre de l'approche communicative s'explique sans doute par l'hétérogénéité des apports théoriques. Rappelons cependant que les théories sont intégrées de manière variable et à différents niveaux. Il reste vrai que certains lignes de force se dégagent par rapport à ces apports théoriques: enseigner une compétence de communication, appréhender le discours dans sa dimension globale, privilégier le sens. (Bérard, 1991: 30).

3 "La finalidad que se persigue en este área es aprender a comunicarse en lengua extranjera, favoreciendo su uso instrumental, lo que implica adoptar un enfoque basado en la comunicación y en el desarrollo progresivo de la competencia comunicativa. Esta no se limita a los aspectos lingüísticos, sino que se extiende a todos los factores que conforman la comunicación. La competencia comunicativa se compone a su vez de: competencia lingüística, competencia sociolingüística, competencia discursiva, competencia estratégica, competencia sociocultural". (Junta de Andalucía, 1995: 8). 
enfoque comunicativo, a la hora de la necesaria revisión de la enseñanza de una lengua extranjera. La enseñanza del español en Francia, a pesar de su rigidez metodológica y del excesivo hincapié en el "écrit oralisé" establece con rigor la progresión en el aprendizaje a lo largo de los cursos, cuida la selección de textos y expone sus objetivos con gran sencillez, lo que merece nuestra atención como modelo alternativo.

\section{YA NO HAY QUE CONOCER LA LENGUA}

En las simplificaciones a las que se ha llegado en la era comunicativa suele asumirse que no hay que conocer la lengua sino su uso. Se ha defendido con contundencia que era necesario dejar de prestarle atención a la descripción de la lengua para dedicar este valioso tiempo a fomentar su uso en la clase de francés. De este modo, se ha instado a abandonar la gramática explícita y los ejercicios "descontextualizados" que servían para afianzar sus reglas, para basar la docencia, a menudo, en ambiguas situaciones de comunicación. Sin embargo, en la ESO, al menos, no se ha logrado en realidad convencer a los profesores de que basen su enseñanza en el uso de la lengua, -sobre todo porque no se sentían capaces de manejar el idioma con seguridad-. Es fácil constatar que buena parte del profesorado sigue dedicando sus clases a lectura y traducción del texto; explicaciones gramaticales superficiales y ejercicios en exceso fáciles que se encuentran en un manual pensado para otro tipo de enseñanza. Pero sin la convicción de que ésta sea la mejor manera de enseñar y, por lo tanto, sin profundizar en dichas explicaciones y actividades. Además, una hipocresía generalizada reina en las planificaciones de aula que se ajustan "a rajatabla" al enfoque comunicativo. Al ser observadas sus clases, muchos de los profesores admiten de buen grado que deberían hablar y escribir más en francés y siempre encuentran excusas que justifican, en su caso, la imposibilidad de poner en práctica sus objetivos ${ }^{4}$. Al hablarles de la necesidad o exigencia de crear una situación de comunicación responden con una sonrisa.

Frente a tales creencias y tales usos, los profesores de español como lengua extranjera defienden abiertamente que su objetivo es la enseñanza de la lengua:

L'enseignement de la langue est et doit rester l'objectif primordial du cours d'espagnol.

C'est lui, et lui seul, qui justifie la place de la langue comme discipline scolaire.

(Bedel et alii, 1996: 14).

\section{Aprender la lengua en situación}

Las pedagogías de la enseñanza del francés y del español vuelven a diferir en su concepción de "situacion" y uso del documento en la clase:

\footnotetext{
${ }^{4}$ Aun creyendo en la necesidad de describir el uso de la lengua, encuentra el doble problema de no hallar en el manual una correcta explicación gramatical ni en los alumnos los suficientes conocimientos sobre lengua materna que faciliten las explicaciones.
} 
...nous entendons par "cours" le cours d'espagnol habituel, pendant lequel la classe étudie un document authentique : texte, document iconographique, séquence de film, etc. (...)

L'étude d'un document crée cette "situation". L'apprenant va manier la langue "en situation", l'enseignant va la faire appréhender "en situation". Ce concept de situation implique une position individuelle dans un contexte et par rapport à un objet. Le contexte est évidemment l'examen du document que constitue le cours ; quant à l' objet, il nous apparaît multiple :

- Il s'agit tout d'abord du document étudié, par rapport auquel se situent l'élève et le professeur (...)

- Ce commentaire constitue lui-même un deuxième objet, le texte produit par un élève déterminé venant, pour quelques instants, se substituer en tant que référent au document étudié. (...)

- Le troisième objet de la situation de classe est -on l'aura compris- le discours du professeur lui-même. (Bedel et alii:1996, 22-23).

Los profesores de español entienden por "situación" únicamente el contexto de la clase en el que se analiza el documento, lo que difiere notablemente de los diversos conceptos que se esconden bajo el mismo término en el enfoque comunicativo que se sigue en España 5 .

En nuestro país hemos adoptado para la enseñanza de la Lengua Extranjera una curiosa estrategia basada en el ilusionismo y la simulación teatral: trasladar mentalmente la clase entera a Francia y transformarla en mercado, aeropuerto, excursión, restaurante o nave espacial parece ser la misión del profesor, ahora conductor de juego. Idea no nueva por otra parte, y que implica, si se quieren obtener resultados, un ímprobo trabajo por parte del enseñante, que sólo en plena juventud y forma es capaz de bajar de la nave en $3^{\circ}$ curso, asistir a una cena en $4^{\circ}$ y al final de la mañana convertir a $2^{\circ}$ en le marché aux puces. Se pretende trasladar a otro país y a una clase la situación de intercambio verbal que produce la adquisición de la lengua materna y, de esa manera, recrear un aprendizaje natural e intencionado. La dificultad

5 "El conocimiento de esa lengua significará aprender de forma apropiada los actos del lenguaje que intervienen en las situaciones de comunicación. Estas situaciones tendrán que ver con la experiencia tanto vivencial como lingüística del alumno, ya que el "conocimiento compartido" facilitará la relación con conceptos existentes, así como la comprensión del sentido de la comunicación”. (MEC, 1992: 14).

"El objetivo de secuenciar los contenidos conceptuales en bloques o áreas de comunicación es el de crear contextos situacionales y promover procesos de interacción en el aula: comprensión, negociación. La elección de las áreas o situaciones de comunicación como unidad de secuencia de contenidos la hemos tomado porque:

a. son representativas de los procesos de comunicación en la vida real.

b. podrían permitir la estructuración de Unidades Didácticas orientadas hacia la realización de una tarea final". (MEC, 1992: 50-51).

"Por otra parte, la comunicación propia del aula en muchos casos no es comunicación real porque el maestro hace preguntas cuya respuesta ya conoce y no se produce la diferencia de información entre los interlocutores que es la característica principal de una comunicación auténtica. Por todo esto es fundamental que el maestro sea consciente de que debe haber un equilibrio entre la comunicación a veces simulada del aula y las situaciones de comunicación más cercanas al mundo exterior, en otras palabras, al alumno se le deben ofrecer muchas oportunidades para mantener una interacción oral que se parezca al máximo a la que se da entre hablantes de la lengua estudiada". (MEC, 1992: 94). 
o el fracaso de este constante cambio es evidente, al no poder reproducirse las mismas condiciones y, por lo tanto, la simulación estar tremendamente forzada. Desde la Pragmática se puede demostrar fácilmente que la verdadera intención comunicativa no se llega a producir en las clases sino en contadísimas ocasiones y que el esfuerzo del profesor por promover, establecer, activar una comunicación simulada suele terminar en gasto inútil de fuerzas.

Se supone que en estas situaciones recreadas los alumnos deben, al mismo tiempo, llegar a "comunicarse" de manera auténtica sin dejar de tener en cuenta la situación de aprendizaje de la que deben hacerse responsables. (La seule authentique "hic et nunc", que decía Ch. Puren). Pero, si es difícil hacer que los alumnos se responsabilicen, convencerlos de que, además, adopten otros papeles para mejorar su dominio del francés lo es mucho más. Algunos profesores se sienten tentados por llevar a cabo tales experiencias. Los más, tras algún que otro intento parcial, acaban por adoptar su "metodología ecléctica" en donde se pueden encontrar magníficas adaptaciones metodológicas y también, en los tiempos que corren, un dejarse llevar al son que toca el libro y seguir aquella rutina que permite tener la clase algo más callada y algo más en orden.

El seguimiento en las aulas de las orientaciones curriculares durante los últimos diez años hace que sea forzoso admitir que la única situación aceptada de la clase de francés sea la propia clase, en la que los alumnos se ejercitan en aprender y reproducir la lengua y no en comunicarse por medio de ella. Ni los diálogos estereotipados de las metodologías audivisuales lograron simular las conversaciones reales, ni las sugerentes iniciativas del enfoque pragmáticocomunicativo han logrado eficazmente recrear la comunicación. Adoptar otra personalidad puede incluso conllevar ciertos desajustes en los alumnos al ser obligatoriamente requeridos a una supuesta situación real de comunicación:

mais il faudra tenir compte du fait que dans ce type d'approches l'apprenant est sollicité en même temps en tant que sujet réel et en tant que sujet imaginaire où il est invité à se projeter et que de ce jeu entre ces deux identités-personnelle et projective- peuvent se dériver non seulement des avantages pour l'apprentissage (le plaisir de satisfaire le désir d'être autre), mais aussi des conflits psychiques inhibiteurs de l'apprentissage (la peur et le refus d'être différent). (Atienza Merino, 2003: 326).

\section{ENFoque discursivo 6}

Siendo este una de las principales aportaciones de la Lingüística a la metodología comunicativa, que deja atrás la frase para adentrarse en el texto y sus intencionalidades, debería inspirar buena parte de las actividades y ser el eje de la organización de contenidos.

${ }^{6}$ Tanto en las Orientaciones didácticas para Primaria como en las de Secundaría se adopta el llamado enfoque discursivo: "Si partimos de la concepción de que la lengua se aprende globalmente y que progresivamente se va refinando y analizando, es decir, que se va del todo a las partes; esto supone que no se debe empezar por la oración aislada, sino por textos o discursos completos aunque estos sean breves."(MEC, 1992: 97).

"La progresión textual, relacionada con el documento o texto que se selecciona, como ilustrativo de la situación de comunicación y en conexión con la misma. La progresión textual viene impuesta a su vez por la progresión del discurso. Progresión textual y discursiva se dan al unísono" (Junta de Andalucía, 1995: 156). 
No obstante, las pizarras de las clases siguen estando llenas de frases, sólo frases; en las anotaciones de los profesores para calificar no suele aparecer la expresión oral -que se confunde a menudo con pronunciación- y raros son los exámenes en los que predominan las preguntas que exigen elaborar un texto, rellenar los huecos de un texto, escribir un comentario, una carta, un diálogo, un resumen...

En la pédagogie de l'espagnol se sostiene que el eje de progresión es explícitamente el uso discursivo de la lengua, el cual no se limita a la simple comprensión del documento sino que se centra en el comentario oral que el alumno realiza. El documento crea, pues, la situación y por ello se extrema el rigor en su elección: la calidad del documento está en función de las posibilidades de expresión que ofrece; su adecuación, en aquel nivel de exigencia que permita una correcta progresión. Proponen una variedad de documentos, no una gran cantidad, en la que alternen los escritos con los visuales cuyo fin sea favorecer el reempleo necesario para su apropiación y memorización.

En este aspecto también difieren del "input comprensible", que ofrece la mayor parte de los manuales de enfoque comunicativo usados en España, cuyos textos se agrupan temáticamente proporcionando un léxico acorde con las situaciones de comunicación. Sin embargo, su utilidad suele verse reducida a satisfacer una rápida comprensión, ornamentada, a menudo, con preguntas de verdadero o falso, que tanto juego dan en la clase y que tan poco útiles son. La pretensión, además, de que estos documentos comunicativos responden a situaciones cercanas y que, por ello, serán mejor acogidos es más que dudosa ${ }^{7}$. No es extraño oír las quejas de los alumnos ante la insistencia de repetir todos los años los mismos temas y la añoranza de los profesores de otros textos con los que se podía sorprender y hacer admirar la lengua.

El Diseño curricular y las Orientaciones para la Secuenciación de Contenidos en el Área de Lengua extranjera de Secundaria establecen, con mucho acierto, que son los contenidos procedimentales los que deben guiar la enseñanza de la lengua. A pesar de ello, es fácil constatar que el dominio de la lengua extranjera que tienen los alumnos en Secundaria y Bachillerato es bastante pobre en general; por lo que, al comparar lo grueso de las guías docentes y la escasez de lo adquirido, nos preguntamos si entre tanto término didáctico no se diluye la adecuación de los contenidos al nivel de exigencia en el aprendizaje a una determinada edad. ¿No se debería haber diferenciado claramente lo que los alumnos son ya capaces de hacer de lo que tienen aún que dominar? Y digo esto porque, si bien se afirma en dichas publicaciones que: "Los procedimientos constituyen el contenido organizador de la secuencia como eje de la actividad de enseñanza y aprendizaje al ofrecer la posibilidad de adquirir el concepto y el proceso requerido para aprender", la mayor parte de dichos procedimientos $^{8}$ son tan evidentes que suelen dejarse de lado, o son simplemente recordados a la hora de pedir un poco de atención a los textos. Por el contrario, algunos de ellos -los

${ }^{7}$ Cette adaptation thématique aux prétendus intérêts de la classe a montré ses limites dans le passé en démontrant, s'il en était besoin, que l'intérêt d'un document ne réside pas-ou du moins pas seulement-dans le thème traité. C'est bien plutôt la lassitude des élèves qu'il faudra s'efforcer d'éviter. (Bedel et alii, 1996: 23).

${ }^{8}$ Como ejemplo: "Anticipar el contenido de un texto oral a partir de indicios textuales, paratextuales o icónicos; hacer suposiciones o anticipaciones de sentido con respecto a lo visto u oído con anterioridad; utilizar formas y modelos lingüísticos antes oídos; hacer suposiciones y reconocer el destinatario de un texto escrito; contextualizar un enunciado analizando la situación de comunicación.” (Junta de Andalucía, 1995: 151). 
que ocupan los últimos lugares- sí suponen un mayor nivel de análisis discursivo, indispensable a nuestro entender, para dominar cualquier lengua ${ }^{9}$ y adecuados, además, a la etapa educativa para la que han sido formulados. Sin embargo, son éstos los que suelen ser relegados por una mayoría de profesores que, amparados en las citadas orientaciones y, sobre todo, en los manuales que las interpretan, no suelen trabajar en su clase aquellos que suponen mayor esfuerzo.

En las ejemplificaciones que acompañan las mencionadas publicaciones oficiales, no se encuentran textos que ofrezcan facilidades para el desarrollo de estos procedimientos propios del Área y de la maduración del alumno. Y por el contrario abundan las actividades que poco o nada le exigen a este. Asímismo, los manuales correspondientes a este nivel están repletos de textos demasiado obvios que no favorecen el comentario o la interpretación y cuyas dificultades lingüísticas suelen ser definitivamente subsanadas en clase mediante una rápida y "concluyente" traducción. Las producciones discursivas de los alumnos escasean igual o más que antes.

\section{LA NUEVA CENSURA DE LOS TEXTOS}

Para el profesor o el simple conocedor de la cultura francesa resulta decepcionante comprobar que la selección de textos que se puede encontrar en los manuales de Secundaria, e incluso de Bachillerato, se halla sometida al encasillamiento de "las situaciones comunicativas de la vida real" y, como resultado, estos documentos, de estilo "simplón", quedan a menudo obsoletos; la información que aportan es escasa y su interés bastante sesgado.

A fuerza de querer cumplir con todo, de atender a la necesidad de una completa formación del alumno en valores y comportamientos, se ha logrado condicionar y desvirtuar algo tan amoldable y al mismo tiempo inabarcable como lo cultural. Por una parte, se ha identificado muy superficialmente la posible motivación del estudiante de francés. Por otra, se han extrapolado a todos los niveles de enseñanza las teorías de psicología del aprendizaje que defendían, en Educación Infantil y Primaria, la mayor facilidad en aprender lo que se sitúa en el entorno próximo del alumno.

En ese intento de cuadratura del círculo nos llama la atención la acepción que de lo cultural $^{10}$ se ha llegado a adoptar en la metodología comunicativa. Distinción bastante discutible

9 "Reconocer las intenciones del locutor; captar la organización global del lenguaje comprendiendo el enlace de ideas y hechos según la lógica discursiva; comprender la idea principal y los detalles del mensaje; hacerse comprender organizando las ideas, formulando la idea principal y los detalles; dar un juicio sobre lo leído y hacer actividades de expresión oral a partir del texto; tomar posición como locutor o escritor; describir una realidad presente, ausente o imaginaria". (Junta de Andalucía, 1995: 151-154).

10 "Se entiende por "cultural" lo referente a la vida cotidiana del mundo francés, mientras que lo "cultivado" hace referencia a lo que tradicionalmente se ha llamado cultura (historia, literatura, artes, ciencias del país) es decir el capital cultural. Trabajar "lo cultivado" sin pasar por "lo cultural" sería alterar la progresión necesaria que, por una parte, respeta los esquemas cognitivos de los alumnos, y por otra, dentro de un aprendizaje comunicativo de la lengua extranjera, no se puede llegar a "lo cultivado" sin pasar por "lo cultural". (Junta de Andalucía, 1995:160-161). 
que lleva a una exclusión o, al menos, a la postposición de algunos temas respecto de otros. Al parecer, la selección de textos se limita en los primeros cursos a esa cultura de la vida cotidiana del mundo juvenil francés. Nueva restricción que igual que otras, a menudo criticadas, han ido limitando la visión de Francia y creando los estereotipos de lo francés. Ahora, desde el primer curso de Secundaria hasta los temas a los que deben ceñirse las pruebas de Selectividad se satura a profesores y alumnos de hábitos alimenticios, uso de ordenadores, telefonía móvil, problemas de medio ambiente, de integración, del tabaco o de la droga, de fracaso escolar. Y si efectivamente aparecen reconocidos textos de literatura ${ }^{11}$, buenas canciones y poemas, sólo constituyen la siempre prescindible guinda del pastel, que se pone al final de la lección, y que no suele "entrar" en la evaluación de los contenidos. Quizás se haya considerado que la clase de francés era el cajón de sastre en donde se podía justificar el tratamiento de los temas transversales: coeducación, salud, consumo, conservación y medio ambiente, democracia y paz; temas aptos, al parecer, para engendrar los actos de habla y garantizar una animada comunicación. Haciendo no aptos aquellos que dejaran de transmitir valores ciudadanos y que son, sin embargo, los que han dado un valor real a la literatura, la política, la canción, el arte o el cine. La cultura a fin de cuentas.

Los profesores de español como lengua extranjera mantienen un enfoque muy distinto al nuestro aunque pequen, quizás, del exceso contrario, que ellos mismos reconocen:

Quant aux réticences qui se font jour ici ou là sur le caractère artificiel, ou trop "littéraire" de l'espagnol des manuels scolaires, il convient d'envisager le problème par rapport à nos objectifs pédagogiques. Rappelons une évidence: la langue apprise et maniée dans la situation artificielle du cours ne peut être celle de la rue, faite d'impératifs et de dialogues que l'on a tant de mal à faire utiliser en classe. Changer de méthode, privilégier l'étude et la restitution du dialogue courant, ce serait accorder à la langue pure une exclusivité pédagogique au détriment de tout l'aspect culturel, éducatif et formateur de notre enseignement. Admettons donc, en restant réalistes, que notre but scientifique ne peut que difficilement, dans la plupart des cas, viser au-delà d'une appréhension des mécanismes linguistiques élémentaires. C'est déjà une base non négligeable qui pourra servir de tremplin pour un approfondissement ultérieur, si le besoin s'en fait sentir... Mais dans un tel système d'apprentissage, la richesse du texte est indispensable, et c'est cette nécessité qui impose, souvent, la langue littéraire.

La richesse de la langue est un élément important, certes, mais non suffisant, de la richesse du texte. Dans notre perspective d'enseignants, celui-ci est riche, essentiellement, des possibilités d'expression qu'il offre, c'est-à-dire de ce qu'il ne dit pas mais laisse entendre. C'est ce non-dit qui soutiendra l'explication et le commentaire de la classe en permettant, en même temps, l'élucidation et la compréhension de la langue du texte, c'est à dire son réemploi ultérieur (...) c'est

${ }^{11}$ Siguiendo estos criterios pocos textos escapan a la férrea censura del prohibidísismo passé simple o la inclusión de subordinadas. 
la raison pour laquelle nombre de textes journalistiques, trop explicites, se prêtent mal au commentaire de classe (...) Au contraire, les textes littéraires sont souvent -mais pas toujours- de bons supports pour l'expression et l'apprentissage de la langue. (Bédel, 1996: 36-37).

Defienden, contra corriente, una metodología basada en textos no inmediatos, llenos de sugerentes posibilidades de comentario. Desechan la utilización de textos periodísticos, bestsellers de nuestro enfoque comunicativo, así como los documentos "branchés directement en prise sur leurs centres d'intérêts et les problèmes des jeunes” puesto que ya han comprobado que los jóvenes se cansaban rápidamente de este tipo de documentos ya que "après tout ils traitaient les mêmes thèmes en cours d'anglais et de français...” (Bédel, 1996: 41).

\section{LA ENSEÑANZA DEL VOCABULARIO}

Es, a nuestro juicio, una de las áreas más perjudicadas con la adopción de los conceptos minimalistas de situación de comunicación y actos de habla: "La adquisición del léxico se produce de forma natural si va unida a las intenciones de los alumnos y a las tareas que estos realizan en contexto comunicativo"; "Los contenidos lexicales que exponemos guardan coherencia con las situaciones definidas y los conceptos pragmalingüísticos y socioculturales en ellas propuestos" (Junta de Andalucía, 1995: 157). Una presumible amplitud debería acompañar a un enfoque que respondiera a las necesidades comunicativas de cada uno de los alumnos ante una situación creada, y, sin embargo, el desarrollo ordenado de una clase eficaz debe someterse a una selección de vocabulario que suele ser presentada en el texto base y en aclaraciones o comentarios del profesor. Si aquel y estos son pobres, el léxico también lo será. Toda la intención comunicativa quedará reducida a los pocos y escogidos términos de los consabidos centros de interés. Su aprendizaje, igual que hacía la metodología audiovisual, a las pocas frases que el alumno es capaz de producir ${ }^{12}$ en las artificiales situaciones de comunicación, sin posibilidad de independizarse de los actos de habla básicos: presentarse, pedir y dar información, localizar, expresar gustos y preferencias, etc.

Siendo más realistas y, a la vez más exigentes, consideramos que en la época del Internet en la que se facilita el acceso a documentos en LE y se puede disponer de traducciones y diccionarios on-line, se debería exigir su lectura y consulta, lo que redundaría en un mayor dominio léxico. Y sin embargo, el vocabulario que ahora tienen los alumnos es menor que el de años atrás, como vemos en las pruebas de Selectividad.

Otro de los usos o creencias que se ha convertido en norma es el de la necesidad de hacer lúdico ${ }^{13}$ el aprendizaje, como si se quisiera distraer al alumno de la seriedad y dificultad

12 Frases que son las que entran en el examen.

${ }^{13}$ Lo que en los primeros cursos de Primaria puede ser aceptado, por la edad de los alumnos, parece lastrar toda la enseñanza del francés: "Y esto es más importante precisamente en el aprendizaje de la lengua extranjera que en cualquier otro, ya que el alumno puede no ver una utilidad inmediata en lo que aprende y le será más fácil implicarse si la actividad le es presentada como un juego, con sus reglas (entre ellas la de comunicarse en la lengua extranjera) y con el disfrute consiguiente". (MEC, 1992: 75). 
de aprender una lengua extranjera. No hay planificación de actividades que se precie que no lleve su sopa de letras, crucigrama o emparejamiento mediante flechas. Su resolución parece llenar de satisfacción al alumno que se siente realizado cuando ve al final de la lección que es capaz de rellenar una ficha que indica: "maintenant je sais... (un peu, assez bien, bien)" y el vocabulario correspondiente. Pocas actividades de verdadera reutilización del léxico se programan en los manuales y aunque en la mente de cualquier profesor está la redacción y las traducciones directa e inversa, es mucho más fácil dejarse arrastrar por los modelos que parecen también servir para aprender el vocabulario, puesto que están en el libro de texto. Si en los primeros cursos de Primaria, fijar la ortografía y/o ayudar a concretar los conceptos léxicos mediante definiciones son objetivos que se deben perseguir, en Secundaria no se debería abandonar el contexto discursivo en el que se inserta el léxico, y manejar la complejidad de la lengua tendría que ser la norma del aula.

Parece como si todos quisiéramos volver a la infancia, pues a la hora de incorporar el juego se abandonan los sólidos presupuestos comunicativos que obligaban a un continuo uso de la situación. Las palabras cruzadas o las listas de palabras poco tienen que ver, pero adornan, rellenan y hacen más atractivos los libros de texto que las incorporan. Al fin y al cabo, el objetivo de las editoriales es, por encima de cualquier otro planteamiento, que se compren más libros.

\section{La ENSEÑanZa de LA GRAMÁtica}

Las metodologías de la enseñanza del francés han coqueteado con la gramática pero una tras otra se rinden a la evidencia de que no puede prescindir de ella, pues los profesores y los alumnos, a partir de una cierta edad, reclaman una sistematización de reglas para comprender y retener el uso lingüístico.

En la enseñanza tradicional de la lengua la gramática tenía la consideración de necesaria, aunque no estimada por los alumnos, que se quejaban de un aprendizaje memorístico de normas y excepciones. Más tarde, implícita en las metodologías audio-orales y audio-visuales, parecía ser una tradición anticuada de la que se podía prescindir. En la actualidad sigue siendo tachada de aburrida, quizás por desconocimiento de quienes la enseñan ${ }^{14}$, pero considerada como mal necesario al que hay que consagrar ciertas horas. Y de ello dan cuenta los manuales que se esfuerzan, no tanto en la calidad de sus escuetas explicaciones, sino en la presentación de cuadros sinópticos en los que abundan los colores, negritas, cursivas y toda clase de elementos que hacen grato a la vista lo pretendidamente esencial.

Declarar el amor incondicional a la gramática podría parecer excesivo, pero resaltar algunas de sus virtudes debería ser una acertada actitud en los futuros enseñantes ${ }^{15}$ que, sin

\footnotetext{
${ }^{14}$ En la formación universitaria de maestros y licenciados no debería desaparecer el estudio en profundidad de la Gramática y ser sustituido por revisiones de los aspectos comunicativos de la lengua. Si las diferentes destrezas se pueden perfeccionar en horas de laboratorio y en estancias en Francia, el conocimiento de la gramática tiene su lugar en la Universidad de la mano de profesores especialistas.

${ }^{15} \mathrm{El}$ profesor mal preparado abusa de explicaciones gramaticales poco claras cuando ni domina el uso de la lengua ni la propia gramática, lo que acentúa su fama de aburrida e inútil.
} 
ella, mal podrán enfrentarse al habitual rechazo que provoca en los alumnos. Un buen conocimiento gramatical hace simple y comprensible lo que, de no tenerlo, parece complejo y farragoso.

Las lenguas son prodigiosos sistemas organizados de signos mucho más interesantes para los que las usan o aprenden que cualquier lenguaje de aplicación informática. Si programar para el ordenador en tal o cual sistema se considera esencial, conocer el funcionamiento de la lengua debería desatar pasiones. Por ello es absurdo menospreciar algo tan bien sistematizado como una gramática, o no recurrir al análisis sintáctico para corregir errores habituales de los hispanohablantes. ${ }^{16}$

La gramática no debería ser considerada como un inventario de respuestas correctas, sino como una excelente ordenación del uso de la lengua en la que el alumno halla el cómo y el porqué de una determinada utilización. Conocer la gramática no lleva a dominar el idioma, pero sin aprenderla y utilizar los atajos que proporciona, cuesta mucho más ir almacenando los conocimientos lingüísticos. Para el profesor no nativo es el mejor instrumento que posee, el que le da seguridad en la corrección, el que explica y justifica el uso. Falto de una sólida base gramatical, fracasará fácilmente en su clase. Inseguro, hará que sus explicaciones no sean claras y fomentará, una vez más, el rechazo a la lengua.

\section{ENFERMEDADES INCONFESABLES}

Hasta el momento hemos expuesto aspectos metodológicos que pueden, o no, explicar el fracaso de la enseñanza del francés en Secundaria. Pero no sería honesto dejar de hablar de la causa más directa de los malos resultados de los alumnos que se incorporan a la Universidad: la enseñanza del francés en los primeros años de enseñanza carece, en general, de un profesorado con un buen dominio de la lengua. Problema que no sólo proviene de la insuficiente cualificación de los enseñantes, sino del temor de las Facultades a tener un mayor nivel de exigencia en el acceso que dé como resultado la pérdida de alumnos, ya bien escasos.

Parece asumido y silenciado por todos que, no ya los maestros en ejercicio-diplomados o licenciados en otra especialidad- sino la mayor parte de los futuros maestros especialistas será incapaz de impartir con soltura una clase de francés, en francés ${ }^{17}$. Que no podrán, por ejemplo, improvisar una serie amplia de preguntas, ni leer correctamente un texto; ni tampoco corregir, sin dudar, una intervención libre o una redacción. Así, desprovistos, se limitarán a lo que dignamente sean capaces de hacer y rehuirán la mayor parte de las actividades que impliquen una verdadera comunicación.

La Coordinación del Practicum en una Facultad de Ciencias de la Educación es fuente de información veraz que nos permite conocer el nivel de enseñanza de colegios públicos y

${ }^{16}$ Por ejemplo, ayudaría a evitar la confusión en las transformaciones pronominales del C. D. y el C. I.; a deshacer el equívoco entre el qui y el que en las oraciones de relativo; a recordar la morfosintaxis de los adjetivos posesivos ante la sustitución habitual de leur por son o poder explicar qué significa un partitivo.

${ }^{17}$ Sería necesario que los futuros profesores tuvieran, en términos del MECRL, un nivel similar al B2 para que el desconocimiento del idioma no fuera un impedimento esencial. 
concertados y cómo se desarrolla la docencia en el primer ciclo de Secundaria en los I.E.S. La situación que solemos encontrar es bien lejana de la que teóricamente debería haber: a pesar de las nuevas leyes que dejaban la enseñanza del idioma en manos de un profesorado, pretendidamente especialista, se suele percibir un relajo casi general en la enseñanza del idioma.

Con indudable buena intención se ha instado a regularizar y adaptar las planificaciones de los profesores a los Diseños Curriculares y a las Orientaciones para la Secuenciación de Contenidos, de esta forma se pretendía mejorar la metodología incorporando el enfoque comunicativo a la programación real de la asignatura. Así, los Planes de Centro parecen reflejar esta adaptación con notable éxito y, sin embargo, las planificaciones oficiales de los maestros, fiel reflejo de las de los manuales, se parecen poco a lo que estos hacen en sus aulas. La imagen de una clase altamente participativa en la que domina la comunicación en francés es pura ficción.

La programación de objetivos que aparece en los libros de texto de mayor difusión suele tender a la exageración y dista de corresponderse con lo que, idealmente, se podría conseguir desarrollando las actividades propuestas. Al incorporarla a la clase, toda una serie de factores intervienen y alejan aún más los objetivos que se perseguían de la actividad real de la clase. Lo más grave de todo ello es que tal disimilitud parece asumida por todos, lo que invalida las planificaciones institucionales. Salvo notables excepciones, el profesor, aferrado al libro escogido, que le ha permitido una secuenciación adecuada de objetivos, contenidos y actividades, no habla francés ni hace hablar a los alumnos; no trabaja con textos que provoquen una reflexión temática y lingüística, ni suele de alentar la escritura libre o la corrección de textos breves personales...

La clase suele consagrarse a la realización de sencillos ejercicios mecánicos de léxico y gramática, a la lectura en voz alta y traducción de mediocres textos periodísticos o diálogos didácticos argotizados y a la resolución de sopas de letras y crucigramas, que ni siquiera ya distraen a los estudiantes adolescentes. Clases de tan bajo nivel suelen ser justificadas por el profesor que atribuye a los alumnos la imposibilidad de una metodología participativa: "no lograrían comprender nada si les hablara exclusivamente en francés"; "mis alumnos no son capaces de hacer periódicamente una redacción". "los motivo ${ }^{18}$ con juegos y actividades sencillas". Parece como si los alumnos redujeran su coeficiente intelectual al entrar en la clase, pues sólo necesitan una mínima parte de él para conseguir buenos resultados. Y es que se establece una multiplicación de efectos entre el profesor que no exige -los objetivos de ciclo son tan generales que todo cabe-; los libros, que con su retórica pedagógica exageran las capacidades adquiridas en tal o cual lección: maintenant vous pouvez exprimer une inquiétude; raconter des événements, cuando apenas se ha comprendido y reutilizado una vez una fórmula que lo exprese; y los alumnos, que consiguen tener un respiro entre otras clases, aunque luego critiquen su escasa preparación en Lengua Extranjera.

${ }^{18}$ En defensa de los maestros es necesario admitir que el ambiente de indisciplina generalizado en las clases, los comportamientos inadecuados de alumnos a los que nos les interesa aprender y la falta de respaldo por parte de los padres a los estudios de los hijos provoca un justificado desánimo y una claudicación ante la imposibilidad de enseñar. 


\section{Y OTRAS SOMBRAS: PLURILINGÜISMO Y JERARQUÍA DE LENGUAS}

Se alzan algunas voces que sostienen que el estudio de otras lenguas extranjeras no tiene que someterse a la "peculiaridad instrumental" del inglés. Es más, consideran que deberían establecerse en el aprendizaje de las lenguas varios objetivos y, por lo tanto, diferentes consideraciones para su estudio, incluso para el propio inglés, separando la lengua de transmisión internacional de la lengua de cultura cuya pedagogía diferiría.

No es de extrañar el rechazo de los humanistas frente a la exclusividad de un aprendizaje exclusivo, si bien necesario, de una lengua descontextualizada:

"L'un des scénarios les pires, les plus appauvrissants, serait en effet que nous parlions tous une sous langue de communication, une "LT1", langue de transmission internationale, mais aussi, (...) cet anglais administratif sans auteur et sans oeuvre ; et que toutes les langues de culture y compris l'anglais de Shakespeare, de Virginia Woolf et d'Oxford) ne subsistent que comme des dialectes, à préserver telles des espèces menacées. (...) Et nous parlerions "entre nous" une langue maternelle peu irriguée, un idiome de communication local... (Cassin, 2005: 25)

"D'où l'importance de mettre au centre de l'enseignement l'idée de "la langue de culture", qui peut bien être ancienne ou vivante, poétique ou scientifique, mais qui se distingue en tout cas de "la langue de service" à but purement communicationnel. Ce que enseigne un professeur "de langue", ce ne sont pas les règles d'une communication réussie, mais un certain rapport à l'autre et à l'étrangeté. L'éducation est fondamentalement l'apprentissage de cet écart à soi et aux autres, dont la langue est le lieu. (Rabouin, 2005: 24)

En la Reforma de la enseñanza de finales de los años 80, a pesar de estar en franco declive el francés frente al ya entonces todopoderoso inglés, se consideró que las directrices de dichas arreas curriculares deberían ser semejantes, si no idénticas. Se hablaba de un único idioma extranjero y, objetivos, contenidos y actividades no diferían entre sí. El francés, prácticamente ha desaparecido en la Enseñanza Primaria y se ha abierto camino en la Enseñanza Secundaria como segunda lengua extranjera. Y si las editoriales han dado buena cuenta de esta implantación, no así los documentos oficiales de referencia, que siguen manteniendo un currículo común desde el tercer curso de Primaria hasta el Bachillerato.

En esta misma línea se avanzan sugerencias sobre una división en las lenguas en relación con la mayor o menor importancia concedida a las destrezas: "para comunicarse, en forma oral y escrita, no es necesario hablar o escribir la misma lengua. Se puede hablar o escribir en la propia lengua y comprender la de los demás. Desarrollar las destrezas comprensivas entre lenguas de una misma raíz linguiística, en los niveles iniciales de la escolarización, supone un planteamiento didáctico (aprender a comprender) más pertinente que el que se deriva de la potenciación de las destrezas productivas" (Vez Jeremías, 2004: 28).

Para las lenguas románicas, propone J. M. Vez Jeremías que se concentre el esfuerzo en la recepción, y añade:

La suma de una competencia completa en lenguas anglosajonas y una competencia receptiva en otras dos o tres lenguas de la Unión Europea configura, sin duda, un 
perfil educativo, cultural y profesional mucho más deseable para los ciudadanos europeos que profesen como tales y tengan razones para conjugar, en la práctica, el verbo comunicar en Europa de una manera muy distinta a la actual. (...) Un gran número de europeos -y no una pequeña minoría- deben disponer de la capacidad de comunicarse en varias lenguas europeas desde el respeto a sus diversas culturas y pesos específicos en la plataforma continental de la Unión.

Pero... ¿ ¿se está realmente tomando este camino?, ¿se desea?, ¿se están aportando los medios necesarios?

Parece razonable admitir que los europeos - a escala masiva- no podrán-como no sucede hoy-llegar a leer, escribir y hablar nada más que en un número muy limitado de lenguas comunitarias. Esto implica el hecho de una imposición de selecciones públicas y personales para un abanico de opciones que siempre serán restrictivas y condicionadas por falta de factores de muy distinta naturaleza. (Vez Jeremías, 2004: 33).

Sin dejar de mantener una posición crítica hacia la reducción artificial de los contenidos de la enseñanza de una LE, reconocemos que desligar el aprendizaje del inglés del de otras lenguas extranjeras puede ser beneficioso e incluso aconsejable. Pero más aconsejable aún debería ser aislar el aprendizaje de idiomas de los intereses políticos ${ }^{19}$, económicos o sociales y dejar que el buen sentido y la experiencia de muchos años de formación en LE orienten la elaboración de un buen currículo, organizado desde Primaria hasta la Universidad, que establezca lo que se debe dominar en cada nivel, que forme adecuadamente al profesorado para poder impartirlo, y que exija a las editoriales una mayor seriedad en sus propuestas.

\section{Bibliografía}

Atienza Merino, J.L. (2003). "L'Émergence de l'inconscient dans l'appropriation des langues étrangères". ELA, 131: 326.

Bedel, J.M., Bermejo, C., Blanco, A., Martinau, M.B. (1996). Un regard sur... La pédagogíe de l'espagnol. Nantes: CRPD des pays de La Loire.

Bérard, E. (1991). L'approche communicative. Paris: CLE international.

Bérard, E. (1995). "La grammaire, encore... et l'approche communicative". ELA, 100: 9-20.

Cassin, B. (2005). "Enseigner au moins deux langues". Le magazine littéraire, 438: 25.

Dabène, L. (1984). "Pour une taxonomie des opérations métacommunicatives en classe de langue étrangère". ELA, 55: 39-46.

Puren, Ch. (1995). "La problématique de la centration sur l'apprenant en contexte scolaire". ELA, 100: $129-150$.

Puren, Ch. (1995). "Des méthodologies constituées et de leur mise en question". Le français dans le monde, Enero: 36-41.

Rabouin, D. (2005). “Quelles langues pour l'Europe?”. Le magazine littéraire, 438: 24.

19 "Por mucho que nos cueste admitirlo, o a pesar de que haya a quien le moleste, los contextos de las Lenguas extranjeras, de su enseñanza y su aprendizaje en el medio escolar formal, no solo son de naturaleza lingüística, sociolingüística, curricular... lo son también de índole política. Y lo son en gran medida" (Vez Jeremías, 2004: 20). 
Vez Jeremías, J.M. (2004). "Contexto político de los aprendizajes de lenguas extranjeras en la dimensión europea". ED.UCO. 1. Servicio de publicaciones de la Universidad de Córdoba: 15-37.

Junta de Andalucía. (1995). Área de Lenguas Extranjeras. Sevilla: Consejería de Educación y Ciencia.

Ministerio de Educación y Ciencia. (1992). Área de lenguas extranjeras. Madrid.

Ministerio de Educación y Ciencia. (1992). Propuestas de Secuencias Lenguas Extranjeras. Editorial Escuela Española, S.A. Madrid. 